[Technical Paper]

\title{
Reduction of Thermal Resistance for Chip Test Technology by Using Super Thermal Conductivity Material and Mirror Finished Silicon
}

\author{
Tomoyuki Hatakeyama*, Masaru Ishizuka*, Yoshiro Nakata**, Motohiro Kuji**, Yusuke Hioki*, \\ Shinji Nakagawa*, and Toshio Tomimura*** \\ *Toyama Prefectural University, 5180 Kurokawa, Imizu, Toyama 939-0398, Japan \\ **ASET, 75-1 Onocho, Tsurumiku, Yokohama, Kanagawa 230-0046, Japan \\ ***Kumamoto University, 2-39-1 Kurokami, Kumamoto, Kumamoto 860-8555, Japan
}

(Received August 16, 2010; accepted November 24, 2010)

\begin{abstract}
In recent years, an innovative 3D-integrated circuit has been developed. Furthermore, innovative chip test technology has also been developed whereby the test is conducted without the need to dice the wafer. Chip tests must be performed at various temperatures. The 3D-integrated circuit has a higher heat generation density than a conventional circuit, and so highly efficient cooling technology is required. We selected an indirect spray cooling technology. With indirect spray cooling, there is a material between the heat generating chips and the sprayed coolant. Therefore, to achieve more efficient spray cooling, the thermal resistance of the material and the contact thermal resistance between the material and the chip must be reduced. In this research, we focused on super thermal conductive composites (STC) to reduce the thermal resistance of the material. Measurement results showed that the thermal resistance of STC is very small and the key issue with the spray cooling chip test is the reduction of the contact thermal resistance between the material and the chip surface. We then evaluated the contact thermal resistance of silicon with a mirror-like finish experimentally. We applied a small amount of liquid between silicon wafers with a mirror-like finish and obtained a very small contact thermal resistance of about $0.2 \times 10^{-4} \mathrm{~m}^{2} \mathrm{~K} / \mathrm{W}$.
\end{abstract}

Keywords: Super Thermal Conductive Composite, Thermal Resistance, Thermal Conductivity, 3D-integrated Circuit, Chip Test, Temperature Management

\section{Introduction}

A 3D-integrated circuit has recently been developed. Furthermore, in the near future a chip reliability test will be conducted before a wafer is diced.[1] There are many chips on one wafer, and all the chips generate heat simultaneously. Since the electrical properties of semiconductor devices are very sensitive to temperature, all the chips on one wafer must be kept at the desired temperature if we are to conduct a precise reliability test. To realize this temperature control, each chip has to be cooled separately and this requires an efficient local cooling technique. A 3Dintegrated circuit has a multi-layered structure and the generated heat becomes much larger than that of a conventional chip with a single layer structure. Therefore, the conventional forced convective cooling technique has insufficient cooling capability to maintain the temperature of each chip, and a cooling method that employs latent heat is a possible alternative.

Under such conditions, we consider that a spray cooling method can be employed for temperature control in a chip reliability test. Two spray cooling techniques can be employed when semiconductor chips are tested, namely direct cooling or indirect cooling.[2] Direct cooling is highly efficient because there is no thermal resistance between the cooled surface and the coolant. However, to put it another way, there is no electrical resistance between the cooled surface and the coolant. This means that the coolant must be a dielectric liquid. In this 
research, we focused on the indirect spray cooling method because it can use water. The use of water as a coolant is attractive in terms of cost. With this method there is a solid material between the coolant and the chips. This means that there is both electrical and thermal resistance between the coolant and the chips. Figure 1 shows a schematic of the indirect spray cooling system. In this system, the wafer is attached to the solid material, which acts as a wafer tray. To achieve a more efficient spray cooling system, the thermal resistance between the coolant and the chips should be reduced. We should consider two thermal resistances as seen in Fig. 2. The first is the thermal resistance of the solid material and the second is the contact thermal resistance between the solid and the chips.

In this research, we consider the possibility of reducing these two thermal resistances. If we are to reduce the thermal resistance of the solid, a material with high thermal conductivity is better than one with low thermal conductivity. We chose the super thermal conductivity composite, STC, supplied by Moriya Cutlery Laboratory, Ltd. This material was developed by Moriya Cutlery Laboratory and Shimane Institute for Industrial Technology.[3] STC has a higher thermal conductivity than copper. To reduce the contact thermal resistance between the solid and the chips, we consider the use of a mirror-finished surface. Normally, contact thermal resistance is caused by the asperity of a surface. A reduction in the contact thermal resistance means a reduction in nano- or micro-scale concavity and convexity. Normally, the real attachment area is much smaller than the non-contact area between solid sur-

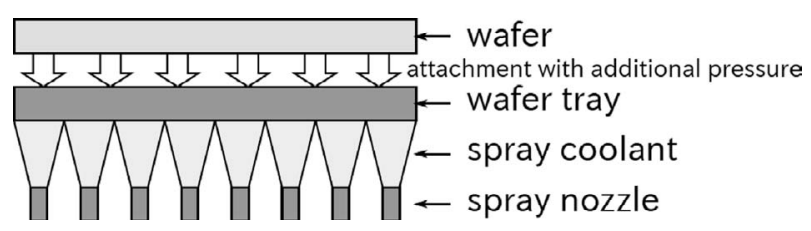

Fig. 1 Image of indirect spray cooling system.

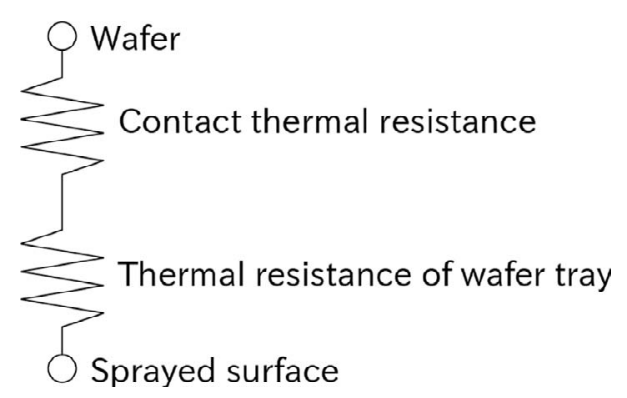

Fig. 2 Significant thermal resistance of indirect spray cooling system. faces. And air fills the nano- or micro-scale space between the solid surfaces. The thermal conductivity of air is much lower than that of metals, and so the contact thermal resistance increases. Hence, the contact thermal resistance can be reduced in two ways. One involves using a larger real contact area and the other is to employ a fluid with higher thermal conductivity between the solid surfaces. Data on the contact thermal resistance between solids have already been published.[4-6] The thermal contact resistance between solid surfaces is less than $10^{-4} \mathrm{~m}^{2} \mathrm{~K} / \mathrm{W}$ with a loading pressure of several MPa.[7] In terms of the chip reliability test, such a high pressure cannot be applied to the wafer. Therefore, we focused on the use of a mirrorfinished surface and employed silicon with a mirror finish. By using mirror-finished silicon, we can increase the real contact area. Furthermore, if air can be replaced with a fluid that has higher thermal conductivity, the contact thermal resistance between solid surfaces can be reduced. Recently, water was applied between a micro machined hydrophilic solid surface and a hydrophobic solid surface and a contact thermal resistance of $5 \times 10^{-5} \mathrm{~m}^{2} \mathrm{~K} / \mathrm{W}$ was realized.[8] However, there are no data for water or another fluid between solid surfaces with complete attachment. Water is particularly attractive because it is easily available and has a relatively high thermal conductivity. Therefore, we filled the small space between the mirrorfinished silicon plates with FC-3283 and water, and measured the thermal resistance at several loading pressures. By using mirror-finished silicon, we were able to measure the thermal resistance between silicon and silicon.

\section{Experimental Apparatus}

Figure 3 shows the balance scale experimental apparatus we used for the thermal resistance measurement.[911] The parts labeled No. 1 and 2 in Fig. 3 are brass bars. Four thermocouples are located on each brass bar at equal intervals as shown in Fig. 4. The brass bars are $40 \mathrm{~mm}$ diameter cylinders with a height of $45 \mathrm{~mm}$. A sample is placed between these brass bars and its thermal resistance

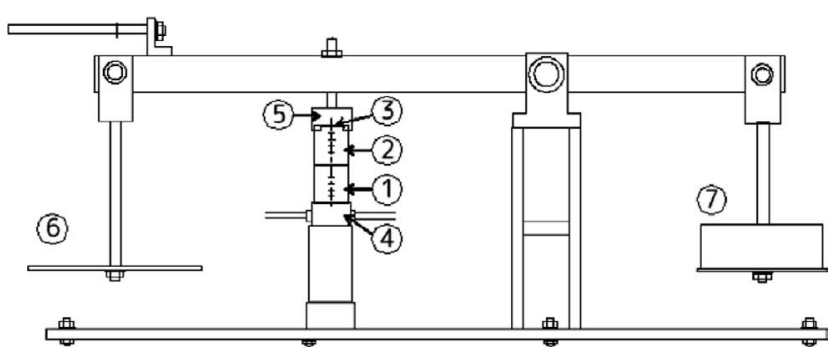

Fig. 3 Experimental apparatus. 
is measured. The part labeled No. 4 in Fig. 3 is a cooling part. Water, which is kept at a constant temperature, flows through this part. A film heater is located at part No. 3 and the heat is generated by a power supply unit. Part No 5 is an acrylic block that insulates the heat flow from the heater to the atmosphere. Part No. 7 is the balancing weight and part No. 6 is the plate for the weight. If no weight is on the plate, the left and right arms are in the balance. If a weight is placed on the plate, a loading pressure is applied to the brass bars. This realizes good attachment between the brass bars and the sample. This system can realize a stable loading pressure. If the loading pressure is controlled electrically, calibration should always be conducted to obtain precise measurements. In the following section, we call the brass bar on the heated side brass bar 2 , and the brass bar on the cooled side brass bar 1 . The procedure for measuring thermal resistance is described below. The generated heat at a constant power flows through the brass bars without any loss since the upper part of the test section is thermally insulated with the acrylic block. If there is no thermal resistance between brass bars 1 and 2, the measured temperature exhibits a linear profile from the heating part to the cooling part. However, the thermal resistance of the measured sample

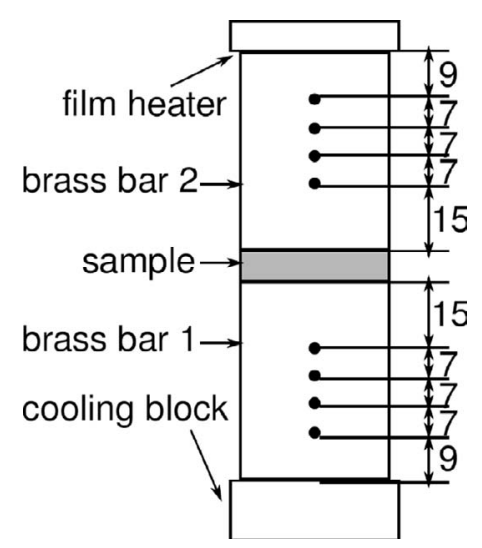

Fig. 4 Location of thermocouples.

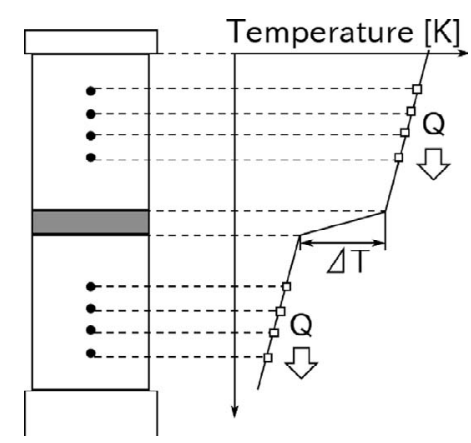

Fig. 5 Experimentally obtained temperature profile. causes a temperature drop between brass bars 2 and 1 . Figure 5 shows an image of the temperature profile in the test section. In each brass bar, the temperature exhibits a linear profile. Since the thermal conductivity of brass has already been reported,[12] heat flowing through the brass bar, $Q[\mathrm{~W}]$, can be calculated. The temperature drop, $\Delta T$ $[\mathrm{K}]$, between brass bars 1 and 2 can be measured experimentally. By using these $Q$ and $\Delta T$ values, the thermal resistance of the sample, $R\left[\mathrm{~m}^{2} \mathrm{~K} / \mathrm{W}\right]$, can be calculated as follows.

$$
R=\frac{\Delta T \cdot A}{Q}
$$

Here, $A\left[\mathrm{~m}^{2}\right]$ is the area where the heat flows.

In this experiment, the supplied power to the film heater is $15 \mathrm{~W}$.

\section{Thermal Properties of STC}

As mentioned above, we chose STC developed by Moriya Cutlery Laboratory and Shimane Institute for Industrial Technology to reduce the thermal resistance of a material.[3] This material has anisotropic thermal conductivity and exhibits a value of $450-630 \mathrm{~W} /(\mathrm{m} \cdot \mathrm{K})$ in the high thermal conductivity direction. In contrast, in the low thermal conductivity direction, the value is $30-45$ $\mathrm{W} /(\mathrm{m} \cdot \mathrm{K}) \cdot[3]$

In this research, the measured sample is a $4 \times 4 \times 4 \mathrm{~cm}^{3}$ cubic block of STC. The STC block is shown in detail in Fig. 6. STC is a composite of graphite and metal, and has anisotropic thermal conductivity as shown in Fig. 6(a). We stacked this material (Fig. 6(b)) and formed a $1 \times 1 \times 4$ $\mathrm{cm}^{3}$ block (Fig. 6(c)). We then bonded this block with adhesive as shown in Fig. 6(d), and finally realized a $4 \times 4 \times$ $4 \mathrm{~cm}^{3}$ block. We used this sample to measure the thermal resistance of the material and to consider the possibility of realizing a spray cooling system.

The thermal resistance of a solid material can be calculated theoretically as follows.[13]

$$
R=\frac{l}{\lambda}
$$

where $\lambda[\mathrm{W} /(\mathrm{m} \cdot \mathrm{K})]$ is the thermal conductivity of the material and $l[\mathrm{~m}]$ is the sample thickness. By using Eq. (2), the thermal resistance of STC becomes $6.35-8.89 \times$ $10^{-5} \mathrm{~m}^{2} \mathrm{~K} / \mathrm{W}$ theoretically in the high thermal conductivity direction.

\section{Results and Discussion for STC}

To consider the reduction of the thermal resistance of the material for indirect spray cooling, we measured the 


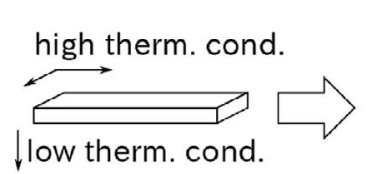

(a)

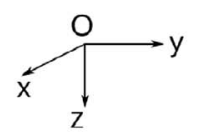

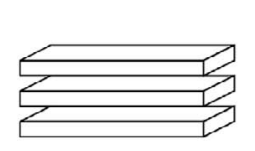

(b)

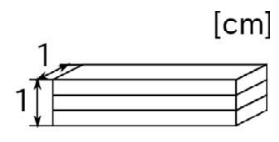

(c)

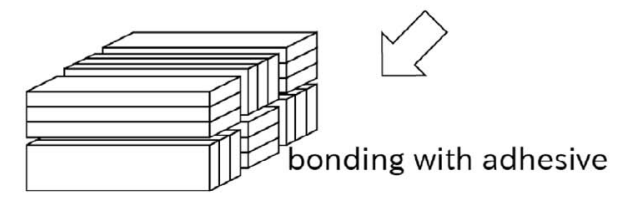

(d)

Fig. 6 Details of STC block.

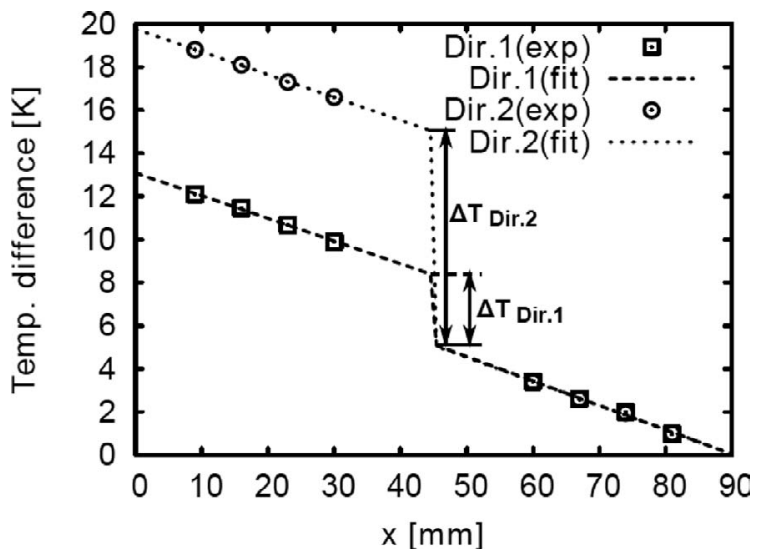

Fig. 7 Measurement results for STC temperature decrease without grease.

thermal resistance of STC. In the experiment described in this section, the pressure between the brass bars and the sample was $78 \mathrm{kPa}$. Since we have no information about the contact thermal resistance between the brass bars and STC, we did not apply thermal conductive grease between the brass bars and STC and considered the effect of the contact thermal resistance.

Figure 7 shows the results of the thermal resistance measurement of STC. In this figure, Dir. 1 and Dir. 2 indicate the high and low thermal conductivity directions, respectively. The points ("exp" in Fig. 7) are the temperatures measured by thermocouples and the lines ("fit" in Fig. 7) are approximated with the least square method. The temperature drop at $\mathrm{x}=45 \mathrm{~mm}$ is caused by the thermal resistance of the measured sample including the contact thermal resistance between the STC and brass bars. The measured thermal resistances are shown in Fig. 8. As seen in the figure, the measured thermal resistance in direction 2 is three times that in direction 1 . However, the thermal resistance in direction 1 is much higher than the theoretical value of $6.35-8.89 \times 10^{-5} \mathrm{~m}^{2} \mathrm{~K} / \mathrm{W}$. This is because of the contact thermal resistance. This result reveals that the contact thermal resistance is significant

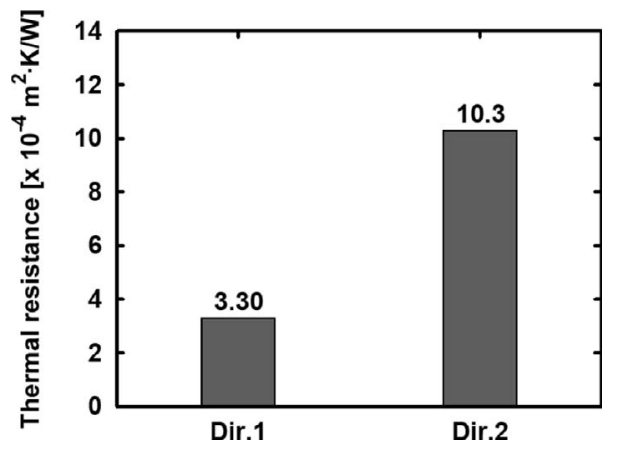

Fig. 8 Thermal resistance of STC without grease.

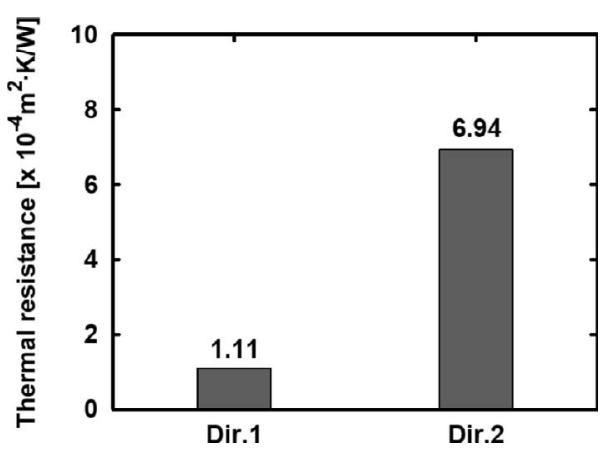

Fig. 9 Thermal resistance of STC with grease.

even if the thermal resistance of the STC is small. By applying STC to the indirect spray cooling technology, the contact thermal resistance should be reduced. Then we applied silicon thermal conductive grease (YG6111, Momentive Performance Materials Japan) between the STC and the brass bars to reduce the contact thermal resistance. The thermal conductivity of this grease is 0.84 $\mathrm{W} /(\mathrm{m} \cdot \mathrm{K})$.

Figure 9 shows the results of the thermal resistance measurement of the STC with the thermal conductive grease. The grease reduces the contact thermal resistance between the brass bars and the sample. As seen in Fig. 9, the measured thermal resistance becomes much lower than the previous case. And the thermal resistance in direction 1 is almost the same as the theoretical value. 
From these results, STC can be applied to the indirect spray cooling system, and a key issue is the reduction of the contact thermal resistance.

In this research, we focus on a chip reliability test. In the spray cooling system, the solid material is part of the test apparatus and the chip is a commercial product. Considering the application, the thermal conductive grease cannot be used because it is a gel and if the grease is applied, the chip surface must be cleaned after the test. Therefore, we have to consider another way of reducing the contact thermal resistance.

\section{Results and Discussion for Mirror Finished Silicon}

In this section, we discuss the reduction of contact thermal resistance. As shown in the previous section, thermal conductive grease can reduce contact thermal resistance. However, gel material cannot be employed. Contact thermal resistance results from the asperity of the surface. A reduction in the contact thermal resistance means a reduction of the effect of nano- or micro-scale concavity and convexity. Here, we focus on mirror-finished silicon. In mirrorfinished silicon, the asperity of the surface is greatly reduced. In this section, we evaluate the contact thermal resistance without the effect of the thermal resistance of the material.

The results of the thermal resistance measurement include the thermal resistance of the material and the contact thermal resistance. To separate these two thermal resistances, we employ the method described below. Figure 10 shows a schematic of the experiment.

In this case, no grease is applied between the brass bar and the silicon plate. Here, we assume that the number of silicon plates is $n$. When we take the thermal resistance of each part into consideration, the measured thermal resis-

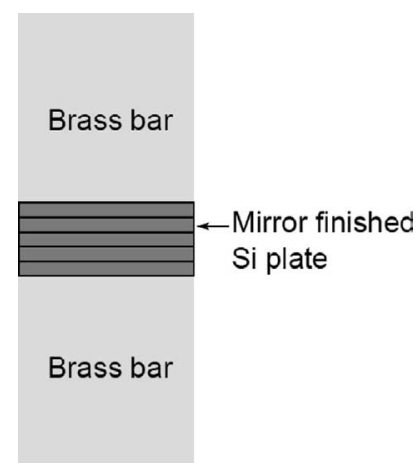

Fig. 10 Schematic of contact thermal resistance measurement. tance can be expressed as below.

$$
R_{\text {total }}=2 R_{\text {si-brass }}+n R_{\text {silicon }}+(n-1) R_{\text {contact }}
$$

Here, $R_{\text {total }}$ is the measured thermal resistance, $R_{\text {si-brass }}$ is the thermal resistance between the brass bar and the silicon, $R_{\text {silicon }}$ is the thermal resistance of the silicon plate, $R_{\text {contact }}$ is the contact thermal resistance between the silicon plates, and $n$ is the number of silicon plates. $R_{\text {total }}$ can be obtained from the measurement. $R_{\text {silicon }}$ can be obtained from Eq. (2). Then the unknown parameters are $R_{\text {si-brass }}$ and $R_{\text {contact }}$. This means that, if measurements are conducted under two conditions, we can obtain two equations and can determine $R_{\text {si-brass }}$ and $R_{\text {contact }}$ In this research, we employed five and ten silicon plates. In both cases, the silicon plates were $4 \times 4 \mathrm{~cm}^{2}$ square and 0.78 mm thick.

As mentioned above, to reduce the contact thermal resistance, the surface asperity must be kept at a small value. When two mirror-finished silicon plates are attached, the attachment between the two plates becomes good and the contact thermal resistance is reduced. In this case, the very small spaces in the concavity and convexity are filled with air. To further reduce the contact thermal resistance, this air should be replaced by a fluid with higher thermal conductivity. Therefore, we applied water and FC-3283 (Fluorinert, 3M) to the surface between the silicon plates. The thermal conductivity of water is $0.61 \mathrm{~W} /$ $(\mathrm{m} \cdot \mathrm{K})$ and that of air is $0.026 \mathrm{~W} /(\mathrm{m} \cdot \mathrm{K})$ under atmospheric pressure and at room temperature.[12] The thermal conductivity of FC-3283 is $0.066 \mathrm{~W} /(\mathrm{m} \cdot \mathrm{K})$.[14] Furthermore, the contact thermal resistance is dependent on the pressure between the silicon plates. We also discuss the pressure dependence of the contact thermal resistance in this section. The loading pressures of $0.016,0.031,0.047,0.062$ and $0.078 \mathrm{MPa}$ are evaluated.

Figures 11-13 show the results of the contact thermal resistance experiments. Figures 11 and 12 show the measured thermal resistance with 5 and 10 silicon plates, respectively. As these figures show, the thermal resistance with air is the highest and that with water is the lowest. This order corresponds to the thermal resistance of fluids. By using these results and Eq. (3), we can obtain the contact thermal resistance between the silicon plates. Figure 13 shows the contact thermal resistance between mirrorfinished silicon plates with fluids. This figure reveals that a lower thermal resistance can be obtained with a higher contact pressure for air and FC-3283. And the contact thermal resistance with water exhibits the lowest value and is 


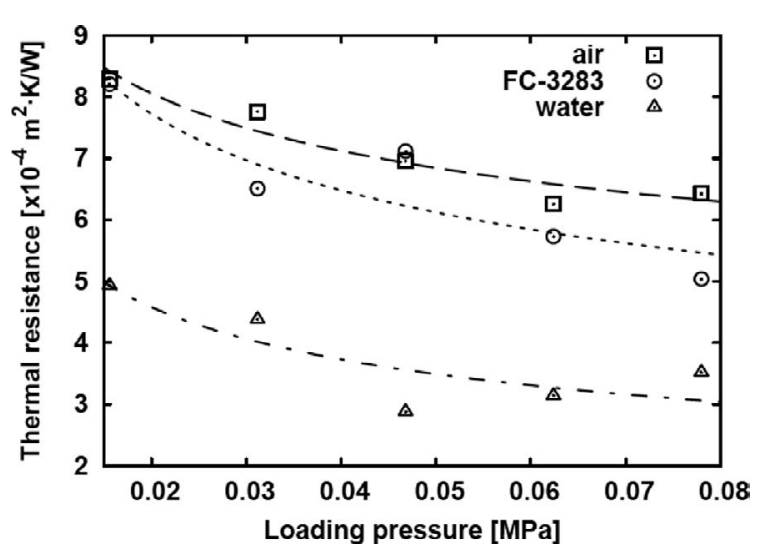

Fig. 11 Measured thermal resistance with 5 silicon plates.

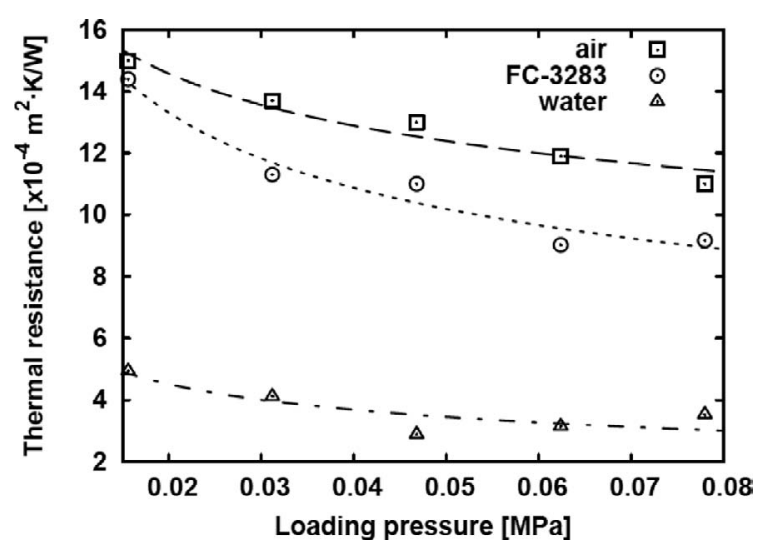

Fig. 12 Measured thermal resistance with 10 silicon plates.

almost independent of the loading pressure. The thermal resistance with water is about $0.2 \times 10^{-4} \mathrm{~m}^{2} \mathrm{~K} / \mathrm{W}$ under any loading pressure.

\section{Evaluation of Overall Thermal Resistance for Application}

The heat flux is $20 \mathrm{~W} / \mathrm{cm}^{2}$ in the 3D-integrated circuit used in this study. Taking this heat flux into account, we evaluate the overall thermal resistance for the chip reliability test application. The thermal resistance of the $4 \times 4 \times 4$ $\mathrm{cm}^{3}$ STC block was $1.11 \times 10^{-4} \mathrm{~m}^{2} \mathrm{~K} / \mathrm{W}$. From Eq. (2), the thermal resistance is halved if the sample thickness is halved. The temperature is measured in the wafer tray shown in Fig. 1 to control the chip surface temperature. Therefore, if the temperature is measured near the chip surface, the thermal resistance of the STC between the temperature measurement point and the chip surface can be greatly reduced. Here, we assume that we measure the temperature $5 \mathrm{~mm}$ from the chip surface. Hence, the thermal resistance of STC becomes $1.39 \times 10^{-5} \mathrm{~m}^{2} \mathrm{~K} / \mathrm{W}$. If the STC surface is mirror finished and water is applied between the STC and the chip, the contact thermal resis-

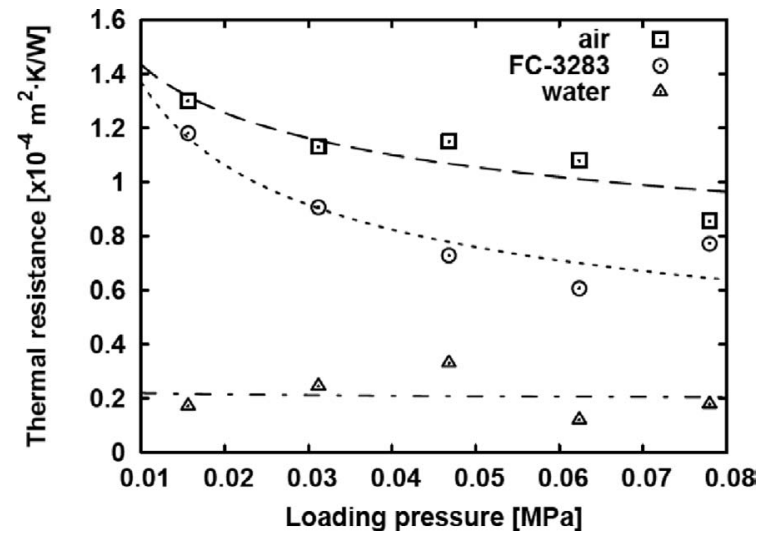

Fig. 13 Contact thermal resistance between $\mathrm{Si}$ plates with fluid.

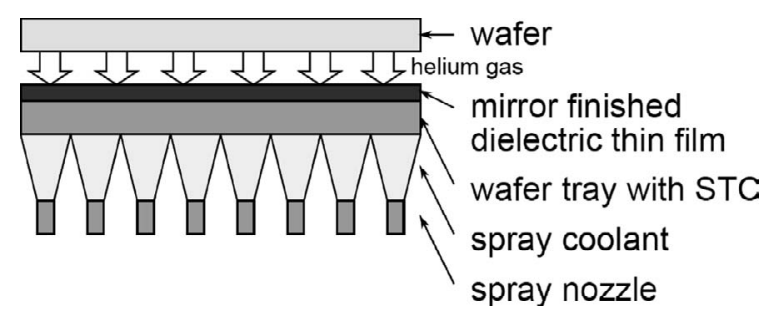

Fig. 14 Application of indirect spray cooling for chip reliability test.

tance may become about $2.0 \times 10^{-5} \mathrm{~m}^{2} \mathrm{~K} / \mathrm{W}$. Therefore, the overall thermal resistance is $3.39 \times 10^{-5} \mathrm{~m}^{2} \mathrm{~K} / \mathrm{W}$. Actually, since the measured thermal resistance of STC includes the thermal resistance of the grease, the overall thermal resistance becomes lower than $3.39 \times 10^{-5} \mathrm{~m}^{2} \mathrm{~K} /$ $\mathrm{W}$. Since the heat flux is $20 \mathrm{~W} / \mathrm{cm}^{2}$, the temperature increase is $6.78^{\circ} \mathrm{C}$ in the present case. For precise temperature control in the chip reliability test, a smaller temperature difference between the temperature measurement point and the chip surface is preferable and the admissible temperature difference is less than $10^{\circ} \mathrm{C}$. If we employ copper as the solid material and apply no fluid between the mirror-finished copper and the chip, the overall thermal resistance becomes $1.09 \times 10^{-4} \mathrm{~m}^{2} \mathrm{~K} / \mathrm{W}$ and the temperature difference becomes $21.8^{\circ} \mathrm{C}$. In this case the loading pressure is about $0.08 \mathrm{MPa}$ as shown in Fig. 13. Therefore, by using an STC, the temperature difference can be reduced below $15^{\circ} \mathrm{C}$ with a loading pressure of $0.016 \mathrm{MPa}$. As a result we can achieve our goal with a lower loading pressure than the conventional technique.

However, we still have certain problems with regard to the application when using STC and water. The electrical resistance of STC is of the same order as copper, and water is an electrically conductive liquid. Therefore, we are considering the application shown in Fig. 14. In this sys- 
tem, since there is a thin dielectric film between the STC and the chip, the electrical conductivity of STC can be neglected. Moreover, the thermal conductivity of helium gas is $0.156 \mathrm{~W} /(\mathrm{m} \cdot \mathrm{K})$ at room temperature.[12] This is one order higher than the thermal conductivity of FC-3283. And helium gas is dielectric. Thus a spray cooling chip reliability test can be achieved.

\section{Conclusion}

We focused on a chip reliability test with an indirect spray cooling technique, and discussed the reduction of the thermal resistance between the chip surface and the coolant. There are two important thermal resistances; one is thermal resistance of a solid material between a chip and a coolant and the other is the contact thermal resistance between a chip surface and a solid material. Based on our thermal resistance measurements, STC can be used to reduce the thermal resistance of a solid material. And to reduce the contact thermal resistance between a chip surface and a solid material, we require good attachment between the two surfaces and must fill the concavity and convexity with a fluid that has a higher thermal conductivity. When water fills the asperities on the mirror-finished silicon plates, the contact thermal resistance becomes approximately $2.0 \times 10^{-5} \mathrm{~m}^{2} \mathrm{~K} / \mathrm{W}$.

\section{Acknowledgement}

This work was entrusted by NEDO "Development of Functionally Innovative 3D-integrated Circuit (Dream Chip) Technology” project.

\section{References}

[1] M. Kada, "Development of Functionally Innovative 3D-Integrated Circuit (Dream Chip) Technology," Proc. of 3DIC 2009, pp. 1-6, 2009.

[2] I. Mudawar, D. Bharathan, K. Kelly, and S. Narumanchi, "Two-Phase Spray Cooling of Hybrid Vehicle Electronics,” Proc. of ITherm2008, pp. 12101221, 2008.

[3] Moriya Cutlery Laboratory, Ltd., http://www.
moriyacl.co.jp/STC/STC.html

[4] Y. S. Ju, M. T. Hung, M. J. Carey, M. C. Cyrille, and J. R. Childress, "Nanoscale Heat Conduction Across Tunnel Junctions,” Applied Physics Letters, Vol. 86, pp. 203113-1-3, 2005.

[5] R. M. Costescu, D. G. Cahill, F. H. Fabreguette, Z. A. Sechrist, and S. M. George, "Ultra-Low Thermal Conductivity in W/Al2O3 Nanolaminates,” Science, Vol. 303, pp. 989-990, 2004.

[6] J. Xu and T. S. Fisher, "Enhancement of Thermal Interface Materials with Carbon Nanotube Arrays," International Journal of Heat and Mass Transfer, Vol. 49, pp. 1658-1666, 2006.

[7] W. B. Song, M. S. Sutton, and J. Talghader, "Thermal Contact Conductance of Actuated Interfaces," Applied Physics Letters, Vol. 81, pp. 1499518-1-3, 2002.

[8] G. Cha and Y. S. Ju, "Reversible Thermal Interfaces Based on Microscale Dielectric Liquid Layers," Applied Physics Letters, Vol. 94, pp. 211904-1-3, 2009.

[9] T. Tomimura, S. Nomura, and M. Okuyama, "Simple Measuring Method of Thermal Conductivity of Silicone Grease and Effect of Carbon Nanomaterials on its Thermal Conductivity," Proc. of HT2007, HT2007-32649, 2007.

[10] Y. Ishida, H. Ryoson, T. Ota, and K. Yazawa, "In-situ, Precise and High-Speed Measurement for Novel TIM Characterization," Proc of IPACK2009, InterPACK2009-89239, 2009.

[11] M. Esmailpour, "Do You Really Know the Thermal Conductivity of Your Boards?," Proc. of $25^{\text {th }}$ IEEE SEMI-THERM Symposium, pp. 55-64, 2009.

[12] JSME ed., JSME Data Book: Heat Transfer $5^{\text {th }}$ Edition, Maruzen Co., 2009.

[13] N. Kunimine, Heat Design Complete Introduction for Electronics (In Japanese), Nikkan Kogyo Shimbun Co., 1997.

[14] 3M, Product Information Fluorinert Electric Liquid FC-3283. 